\title{
CT Scan-Guided Abrams' Needle Pleural Biopsy versus Ultrasound-Assisted Cutting Needle Pleural Biopsy for Diagnosis in Patients with Pleural Effusion: A Randomized, Controlled Trial
}

\author{
Muzaffer Metintas ${ }^{\mathrm{a}}$ Huseyin Yildirim ${ }^{\mathrm{a}}$ Tamer Kaya $^{\mathrm{b}}$ Guntulu Ak $^{\mathrm{a}}$ \\ Emine Dundar $^{c}$ Ragip Ozkan ${ }^{b}$ Selma Metintas ${ }^{d}$ \\ Departments of a Chest Diseases, ${ }^{b}$ Radiology, ${ }^{c}$ Pathology, and ${ }^{d}$ Public Health, Eskisehir Osmangazi University \\ Medical Faculty, Eskisehir, Turkey
}

\section{Key Words}

Pleural disease $\cdot$ Pleural biopsy · Diagnosis

\begin{abstract}
Background: Image-guided pleural biopsies, both using ultrasound (US) or computed tomography (CT), are important in the diagnosis of pleural disease. However, no consensus exists regarding which biopsy needles are appropriate for specific procedures. Objectives: In this randomized, prospective study, we aimed to compare CT scan-guided pleural biopsy using an Abrams' needle (CT-ANPB) with US-assisted pleural biopsy using a cutting needle (US-CNPB) with respect to both diagnostic yield and safety. Methods: Between February 2009 and April 2013, 150 patients with exudative pleural effusion who could not be diagnosed by cytological analysis were included in the study. The patients were randomized into either the US-CNPB group or the CT-ANPB group. The two groups were compared in terms of diagnostic sensitivity and complications. Results: Of the 150 patients enrolled in this study, 45 were diagnosed with malignant mesothelioma, 46 were diagnosed with metastatic pleural dis-
\end{abstract}

ease, 18 were diagnosed with pleural tuberculosis, 34 were diagnosed with benign pleural disease, and 7 were lost to follow-up. In the US-CNPB group, the diagnostic sensitivity was $66.7 \%$, compared with $82.4 \%$ in the CT-ANPB group; the difference between the two groups was statistically significant $(p=0.029)$. The sensitivity of CT-ANPB increased to $93.7 \%$ for patients with a pleural thickness $\geq 1 \mathrm{~cm}$. The complication rates were low and acceptable. Conclusions: The first diagnostic intervention that should be preferred in patients with pleural effusion and associated pleural thickening on a CT scan is CT-ANPB. US-CNPB should be used primarily in cases for which only pleural thickening but no pleural effusion is noted.

(c) 2016 S. Karger AG, Basel

\section{Introduction}

Pleural effusions are often the presenting feature of pleural disease [1]. However, approximately $50 \%$ of all pleural effusion cases remain undiagnosed following an initial diagnostic thoracocentesis $[1,2]$. In the presence

\section{KARGER}

E-Mail karger@karger.com

www.karger.com/res
(C) 2016 S. Karger AG, Basel

$0025-7931 / 16 / 0912-0156 \$ 39.50 / 0$
Prof. Muzaffer Metintas, MD

Department of Chest Diseases, Eskisehir Osmangazi University Medical Faculty Meselik

TR-26040 Eskisehir (Turkey)

E-Mail muzaffermetintas@gmail.com 
of non-diagnostic cytology, medical thoracoscopic and image-guided pleural biopsies are the preferred diagnostic modalities, as both are characterized by a greater diagnostic sensitivity compared with blind pleural biopsy $[3,4]$. However, medical thoracoscopy (MT) requires a degree of expertise and is not available in many parts of the world; therefore, image-guided pleural biopsies are the preferred initial diagnostic procedure $[4$, 5]. Recent studies have proposed that image guidance with either ultrasound (US) or computerized tomography (CT) significantly increases the yield of such biopsies and also decreases the risk of complications $[3,5$, 6]. We performed a prospective study comparing the safety and efficacy of CT scan-guided Abrams' needle pleural biopsy (CT-ANPB) and MT and observed that the two methods did not differ significantly with respect to both diagnostic sensitivity and complication rates [6]. US-assisted pleural biopsy performed using TruCut needles (US-CNPB) has also been utilized frequently [7-9].

In this randomized, prospective study, we aimed to compare CT-ANPB and US-CNPB in terms of their diagnostic yield as the primary endpoint. The secondary endpoint was the safety of both methods.

\section{Materials and Methods}

This prospective, randomized, parallel study was conducted at the Department of Chest Diseases, Eskisehir Osmangazi University, Eskisehir, Turkey, from February 2009 to April 2013. The study was approved by the Ethics Committee of Eskisehir Osmangazi University (2009/91). The clinical approval number (ClinicalTrials.gov identifier) is NCT01196585.

\section{Patients}

Patients with the following criteria were included in the study: evidence of exudative pleural effusion for which a specific diagnosis could not be determined by cytological examination and willingness to participate in the study and to undergo an invasive procedure. Exclusion criteria were the following: age under 18 years, the presence of parapneumonic effusion, the presence of a contraindication for pleural biopsy, or the presence of any other systemic disease which could affect CT or US assessments. The patients were thoroughly informed on the study procedures before randomization, and their written consent was requested.

The patients were randomized into different groups after being hospitalized. The randomization was performed in accordance with the Consolidated Standards of Reporting Trials (CONSORT) statement recommendations. Block randomization with a sequence of 6 was used. The patients in arm A underwent US-CNPB, the patients in arm B underwent CT-ANPB. All patients had CT scans before biopsies.
Ultrasound-Assisted Cutting Needle Pleural Biopsy

Transthoracic US was performed by a senior radiologist (T.K. or R.O.) (Toshiba Just Vision 200 SSA-320A; Toshiba Medical Systems Corporation, Tochigi-ken, Japan). The preferred patient position for the procedure was the sitting position, with the subject's arms folded across the chest and supported by a bedside table. The dorsolateral thoracic wall was surveyed using a standard, 3.75$\mathrm{MHz}$, curvilinear probe. The presence of an effusion was confirmed via standard means $[7,10]$.

During the US scan, all major blood vessels were identified prior to biopsy to avoid injury. Cutting needle biopsies were performed using manually operated 16-gauge Tru-Cut biopsy needles with a specimen notch of $20 \mathrm{~mm}$ (Allegiance, Chateaubriand, France) [7].

If either a pleural mass or nodular, irregular or smooth pleural thickening $>1 \mathrm{~cm}$ with effusion was noted on US, the biopsy was performed at that site. US-CNPB was usually carried out by a free-hand technique without direct US observation. The biopsy site was determined by the US operator who also decided on the direction and depth of penetration. The skin was marked and the biopsy was performed while the patient remained in the same position. If pleural thickening was $<1 \mathrm{~cm}$ with effusion or only pleural effusion without pleural thickening was noted on US, the cutting needle was carefully introduced through the incision site at $45^{\circ}$ to the skin in the direction of the chosen intercostal space above the lower rib to the depth indicated by US [7, 10-14]. If only a pleural effusion was observed, biopsies were performed at the midscapular line with the same technique. For minimal to moderate effusions, the biopsies were performed at the site of the largest effusion, as determined via US. Regarding large effusions, the lowest possible puncture level was chosen, provided that the site was at least $25 \mathrm{~mm}$ from the diaphragm [7, $10,11,15]$. Four to six biopsy specimens were taken from the parietal pleura.

\section{Computerized Tomography Scan-Guided Abrams' Needle} Pleural Biopsy

Contrast-enhanced CT scans of all patients were performed using the Toshiba Aquilla 64 MDBT scanner (Toshiba Medical Systems Corporation). The thorax was scanned at a thickness of $5 \mathrm{~mm}$ from the apex of the lungs to the costophrenic recess. Before the analysis, $80 \mathrm{ml}$ non-ionic contrast substance was administered through an arm vein, and the scan was initiated $30 \mathrm{~s}$ following the infusion of the contrast medium.

The ANPBs were performed in the patients just after determining the entry site with the aid of a CT scan, which was obtained before the biopsy procedure. The entry site for biopsy was selected as the most suitable and accessible part of the lesion by looking at the CT scans on monitors while the patients were in the bronchoscopy room. The distance between the entry site and the target point was measured two-dimensionally by thoracic CT. For instance, the entry site in one patient was $13 \mathrm{~cm}$ away from the bottom edge of the scapula and $7 \mathrm{~cm}$ away from the spinal process of the vertebrae. In another example, the entry site was $0.5 \mathrm{~cm}$ above the carina horizontally and $9 \mathrm{~cm}$ away from the midsternal line laterally. Measurements were made according to the scale located on the CT scans. After taking the measurements described above, the entry site for the Abrams' needle was marked on the skin of the patient as well as the corresponding point for the lesion on the CT scan. If only a pleural effusion was observed on a CT scan, the biopsies 
Table 1. Distribution of the diagnoses of the 150 patients included in this study

\begin{tabular}{lr}
\hline Diagnosis & $\mathrm{n}$ \\
\hline Malignant mesothelioma & 45 \\
Metastatic malignant pleural effusion & 46 \\
Malignant pleural effusions caused by lung cancer & 22 \\
Pleural metastasis due to other organ carcinomatosis & 20 \\
Lymphoma & 4 \\
Tuberculous pleurisy & 18 \\
Benign pleural effusion & 34 \\
$\quad$ Paramalignant pleural effusions & 2 \\
Benign asbestos pleurisy & 11 \\
Rheumatoid pleurisy & 3 \\
Postcardiac injury/cardiac disease & 4 \\
Chronic liver disease & 1 \\
Pulmonary thromboembolism & 2 \\
Indeterminate cases with benign pleural effusion & 11 \\
completely healthy after 2 years & 7 \\
Inadequate follow-up after first invasive intervention & 150 \\
\hline Total number of cases & \\
\hline
\end{tabular}

were performed at the midscapular line [6]. The CT-ANPBs were performed according to a standardized procedure $[6,7]$. Four to six biopsy specimens were taken from the parietal pleura, using the distal tip of the Abrams' needle at a $45^{\circ}$ angle to avoid causing lacerations of the intercostal vessels, through the same entry point, by an experienced pulmonologist (M.M., H.Y., or G.A.) in a bronchoscopy/thoracoscopy room.

Following each procedure, the biopsy specimens were immediately fixed in formalin and sent to the pathology department for histopathological analysis. If suspicion of tuberculous pleurisy was high, an additional biopsy specimen was sent to the laboratory in an isotonic saline solution for bacteriological examination, including a search for Mycobacterium tuberculosis.

In both arms of the study, the patients with a histopathological diagnosis of 'fibrinous pleuritis' underwent MT as well as either video-assisted thoracoscopic surgery or open surgical biopsy, at the patient's discretion. Patients suffering from recurrent symptoms as well as patients with either clinical or radiological signs of disease were re-assessed, and invasive diagnostic procedures were repeated as needed.

Patients with asbestos-related benign pleural disease, tuberculosis, or non-tuberculous benign causes were followed for a minimum follow-up period of 24 months.

\section{Histopathological Analysis}

All biopsy samples were evaluated by the same pathologist (E.D.) of the Department of Pathology, Eskisehir Osmangazi University Medical Faculty, Eskisehir, Turkey. The cases were categorized primarily as benign and malignant, and the malignant cases were subsequently categorized based on cell properties. Immunohistochemical stains were used to distinguish tumors of mesothelial origin from those of epithelial origin. Among these, antibodies, such as carcinoembryonic antigen, Ber Ep4, B 72.3, and CD 15 (Leu-M1), as well as mesothelial cell determinants, such as cal- retinin, Wilm's tumor 1 , thombomodulin, and cytoceratine 5/6, were utilized. Additionally, epithelial membrane antigen, vimentin, p53, and wide-spectrum keratin were used as needed. The pathologist also stained the specimens with Ziehl-Neelsen to test for acid-resistant bacilli (M. tuberculosis).

\section{Statistical Analysis}

Considering that the diagnostic sensitivity was expected to be $65 \%$ for US-CNPB [7] and 87\% for CT-ANPB [6], the estimated minimum sample size needed for this study was 57 patients, with a correction pattern effect of 62 patients in each arm, with a power of $80 \%$ and a significance of $95 \%$. However, we included as many as 78 patients in each arm in case some patients had to be excluded in the course of the study. The primary endpoint of this study was the determination of both the sensitivity and the complication rates of both invasive methods with respect to the diagnosis of pleural disease. SPSS software, version 15.0 (SPSS Inc., Chicago, Ill., USA), was used for the statistical analysis, and the $\chi^{2}$ test and 2 -sided Fisher's exact test were used to compare the groups. The complication rate for both methods was calculated by post-hoc power analysis.

\section{Results}

The total number of patients included in this study was 156. However, 6 patients decided to leave the study during randomization. Therefore, a total of 150 patients were randomized into the two groups. The distribution of the diagnoses of these 150 patients is given in table 1 .

A diagram of the randomization profile of the patients enrolled in this study is shown in figure 1. US-CNPB was performed in 75 patients, including $54(72 \%)$ males and 21 females $(28 \%)$ with a mean age of $61.1 \pm 1.4$ years (range: $29-83$ ). CT-ANPB was performed in 75 patients, including $45(60 \%)$ males and $30(40 \%)$ females with a mean age of $61.7 \pm 1.4$ years (range: $20-82$ ). There were no statistically significant differences in terms of sex $\left(x^{2}=2.406 ; p=0.121\right)$, age $(t=0.294 ; p=0.769)$, or the distribution of diagnoses between the two groups $\left(\chi^{2}=\right.$ 3.880; $\mathrm{p}=0.275$ ).

The proportion of patients with adequate tissue sampling for histopathological examination was $68 / 75$ (90.7\%) in arm A and 77/78 (98.7\%) in arm B. Therefore, successful sampling was performed in more than $90 \%$ of the patients in both groups, with no significant differences noted between the groups $\left(\chi^{2}=0.452 ; \mathrm{p}=0.501\right)$.

There was a significant difference between the two methods with respect to diagnostic sensitivity. The diagnostic sensitivities of the procedures for the patients randomized to the two arms are given in table 2 .

The numbers of biopsies for patients with malignant pleural diseases were 49 and 44 in arm A and arm B, re- 
Table 2. The sensitivity of US-CNPB and CT-ANPB

\begin{tabular}{|c|c|c|c|c|c|}
\hline \multirow[t]{2}{*}{ Diagnosis } & \multicolumn{2}{|l|}{ US-CNPB } & \multicolumn{2}{|l|}{ CT-ANPB } & \\
\hline & $\begin{array}{l}\text { performed, } \\
\mathrm{n} / \mathrm{N}\end{array}$ & $\begin{array}{l}\text { sensitivity, } \\
\%\end{array}$ & $\begin{array}{l}\text { performed, } \\
\mathrm{n} / \mathrm{N}\end{array}$ & $\begin{array}{l}\text { sensitivity, } \\
\%\end{array}$ & \\
\hline All cases & $48 / 72$ & 66.7 & $61 / 74$ & 82.4 & $\chi^{2}=4.794 ; \mathrm{p}=0.029$ \\
\hline All cases of malignancy and pleural tuberculosis & $34 / 55$ & 61.8 & $44 / 56$ & 78.6 & $\chi^{2}=3.728 ; \mathrm{p}=0.054$ \\
\hline
\end{tabular}

Fig. 1. The randomization profile of the pa-

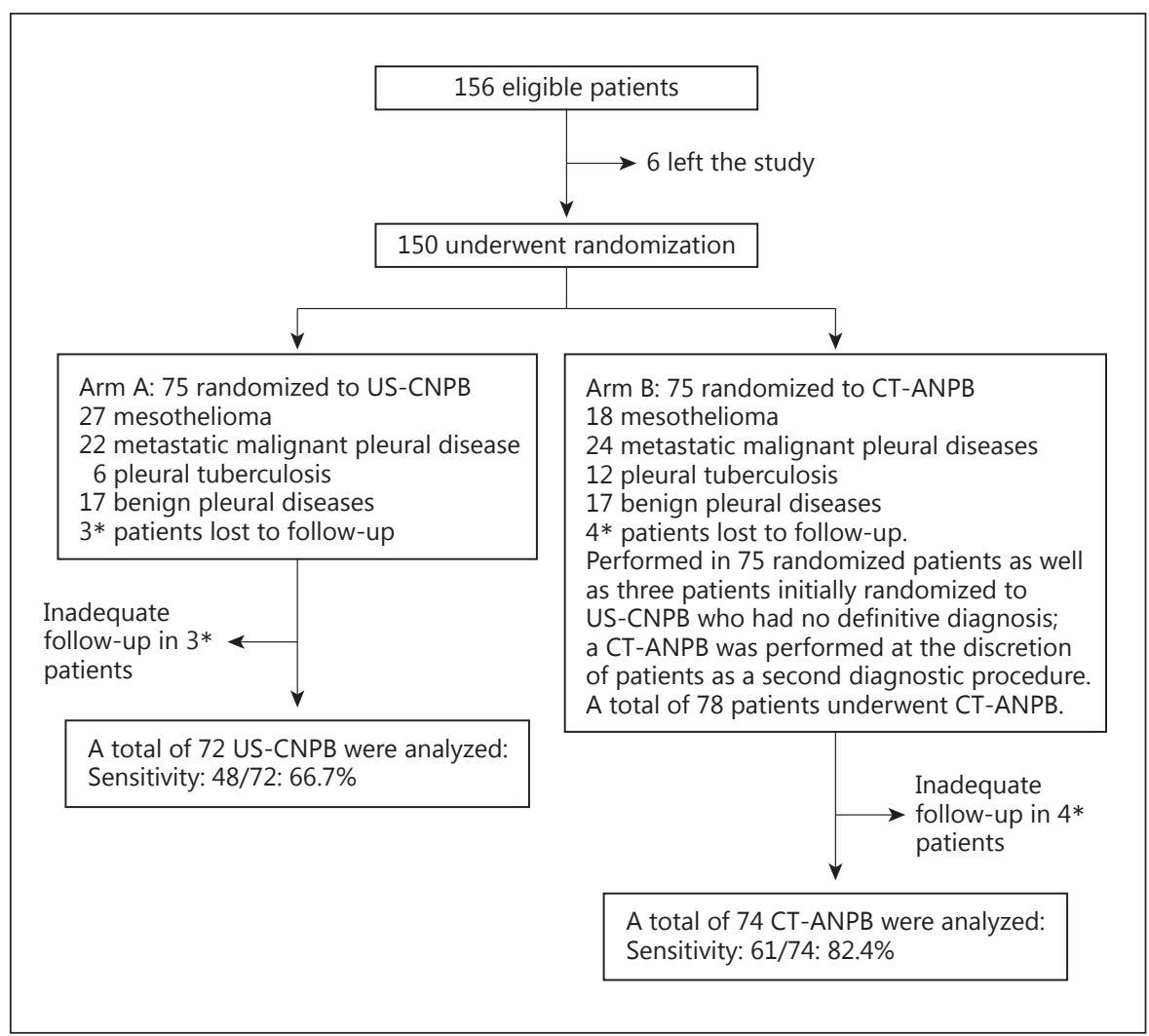

spectively, with diagnostic sensitivities of 61.2 and $77.3 \%$ for US-CNPB and CT-ANPB, respectively. Six patients in arm $A$ and 12 patients in arm B had a diagnosis of pleural tuberculosis. The corresponding diagnostic sensitivities in these two groups were 66.7 and $83.3 \%$, respectively. In arm A, of the 72 patients who underwent a US-CNPB as their initial diagnostic test, 38 had a histopathological diagnosis of 'fibrinous pleuritis.' A final diagnosis of either malignant pleural disease or pleural tuberculosis was made in 21 (55.3\%) of these patients. In arm B, of the 74 patients who initially underwent a CT-ANPB, a histopathological diagnosis of 'fibrinous pleuritis' was report- ed in 29 patients. A final diagnosis of either malignant pleural disease or pleural tuberculosis was made in 11 (37.9\%) of these patients. The difference between the two arms was not statistically significant $\left(\chi^{2}=2.327 ; \mathrm{p}=\right.$ $0.127)$.

Table 3 includes the sensitivity of the two methods with respect to pleural characteristics on a thoracic CT. In patients with a pleural thickness $<1 \mathrm{~cm}$, the diagnostic sensitivities of both modalities were significantly lower than in those with a lesion $\geq 1 \mathrm{~cm}$.

The types and frequencies of the complications for both methods are included in table 4. No major 
Table 3. The sensitivity of the two methods according to the distribution of pleural thickness characteristics observed via $\mathrm{CT}^{1}$

\begin{tabular}{|c|c|c|c|c|}
\hline \multirow{2}{*}{\multicolumn{2}{|c|}{ Biopsy method }} & \multicolumn{2}{|l|}{ Pleural thickness or pleural lesions } & \\
\hline & & \multirow{2}{*}{$\begin{array}{l}<1 \mathrm{~cm} \text {, or only a pleural effusion } \\
12 / 28\end{array}$} & \multicolumn{2}{|l|}{$>1 \mathrm{~cm}$} \\
\hline US-CNPB & $\begin{array}{l}\text { performed, } \mathrm{n} / \mathrm{N} \\
\text { sensitivity, } \%\end{array}$ & & $\begin{array}{l}20 / 25 \\
80.0\end{array}$ & $\chi^{2}=7.616 ; p=0.006$ \\
\hline CT-ANPB & $\begin{array}{l}\text { performed, } \mathrm{n} / \mathrm{N} \\
\text { sensitivity, } \%\end{array}$ & $\begin{array}{l}13 / 23 \\
56.5\end{array}$ & $\begin{array}{l}30 / 32 \\
93.7\end{array}$ & $\chi^{2}=10.873 ; p=0.001$ \\
\hline Total & $\begin{array}{l}\text { performed, } \mathrm{n} / \mathrm{N} \\
\text { sensitivity, } \%\end{array}$ & $\begin{array}{l}25 / 51 \\
49.0\end{array}$ & $\begin{array}{l}50 / 57 \\
87.7\end{array}$ & $\chi^{2}=18.998 ; p=0.001$ \\
\hline
\end{tabular}

${ }^{1}$ This analysis was performed only for malignant pleural effusions and pleural tuberculosis cases.

Table 4. Complications of the diagnostic methods

\begin{tabular}{lll}
\hline Complications & $\begin{array}{l}\text { US-CNPB } \\
(\mathrm{n}=75)\end{array}$ & $\begin{array}{l}\text { CT-ANPB } \\
(\mathrm{n}=78)\end{array}$ \\
\hline Minor bleeding & 1 & 1 \\
Hypotension & 1 & 1 \\
Syncope & 0 & 1 \\
Pain & 2 & 5 \\
Subcutaneous emphysema & 0 & 1 \\
Pneumothorax & 1 & 1 \\
Nausea & 1 & 0 \\
\hline
\end{tabular}

complications occurred in either group. $1-\beta$ was 0.168 for the complication rate. A patient with syncope and hypotension was treated with intravenous physiological saline, and analgesia was administered to patients complaining of pain. The cases who developed bleeding or pneumothorax were successfully treated with rest and oxygen.

\section{Discussion}

We randomly assigned 124 patients with effusions to groups that underwent either a CT-ANPB or MT. In the CT-ANPB group, the diagnostic sensitivity was $87.5 \%$ compared with $94.1 \%$ in the MT group ( $\mathrm{p}=$ 0.252) [6].

Previous, noncomparative studies have reported a higher diagnostic sensitivity using CT-guided techniques as opposed to US-assisted/guided techniques [16, 17]; however, other studies have reported similarly high diag- nostic yields for both techniques [18-21]. It is believed that the choice between US and CT guidance is often influenced by factors such as individual preference, expertise, appliance availability, and cost $[8,20]$. These results suggest that both methods offer certain advantages. However, there exists no consensus regarding the best imaging method with which to guide pleural biopsies, as is the case regarding the type of needle to be used.

To the best of our knowledge, no other studies have examined this issue, with the exception of a single, randomized, comprehensive study comparing US-guided cutting needle biopsy with US-guided Abrams' needle biopsy [7].

One limitation of Abrams' biopsy is related to the blindness of the procedure. However, when a thickened or seemingly problematic pleural zone is located using CT scans, diagnostic sensitivity exceeds $80 \%[6,7,18,22$, 23].

In this study, our aim was to compare US-CNPB with CT-ANPB, the efficacy of which was demonstrated previously by our group, with respect to both diagnostic yield and safety to determine the best approach for specific patient categories, as a means of defining the methods that would ideally be used by radiologists or pulmonologists.

Our decision to utilize US-CNPB in the study's second arm was based on the fact that US is a mobile diagnostic device and may be performed in the bronchoscopy room, as well as the fact that cutting needles were appropriate for performing a real-time biopsy with US [21, 24-29]. Additional support for our decision was provided by the results of the comprehensive, randomized study of Koegelenberg and colleagues, in which the comparison between an Abrams' needle and a cutting 
needle demonstrated the superiority of the former approach [7].

When the overall diagnoses in our patient group were considered, the diagnostic sensitivity of CT-ANPB (82.4\%) was significantly higher than that of US-CNPB (66.7\%). When the diagnostic categories were restricted according to specific diagnoses of either malignant pleural diseases or pleural tuberculosis, the difference in diagnostic yield was $16.8 \%$ (78.6 vs. $61.8 \%$; $p=0.054$ ). These results suggest that, among patients with pleural effusions, CT-ANPB exhibited a higher diagnostic sensitivity compared with US-CNPB. In the study by Koegelenberg et al. [7], Abrams' needle biopsies exhibited a diagnostic yield of $78.7 \%$ compared with $62.9 \%$ for the Tru-Cut needles ( $\mathrm{p} \otimes 0.014)$. Pleural tuberculosis was diagnosed in 66 patients. Abrams' needle biopsies yielded histological confirmation in $83.3 \%$ of these patients, and Tru-Cut needle biopsies yielded histological confirmation in $60.6 \%$ of these patients. Malignant pleural effusions were diagnosed in 12 patients. Abrams' needle biopsies yielded histological confirmation in $10(83.3 \%)$ of these patients, and Tru-Cut needle biopsies yielded histological confirmation in $8(66.7 \%)$ of these patients [7]. In another study, the reported diagnostic yield for US-guided biopsy was $70 \%$ [16]. A recent study noted that the diagnostic sensitivity of Tru-Cut needles was $76.5 \%$ for tuberculous pleurisy and $54 \%$ for malignant pathologies, with an overall sensitivity of $61.1 \%$ [27].

In our study, the histopathological sensitivity of the biopsies performed with a Tru-Cut needle was $66.7 \%$ overall, $61.8 \%$ for malignant pleural diseases and pleural tuberculosis, $59.3 \%$ for mesothelioma, $63.6 \%$ for metastatic malignant pleural disease, and $66.7 \%$ for pleural tuberculosis. Besides both our study and the prospective and randomized study performed by Koegelenberg et al., additional nonrandomized studies also reported higher numerical values with respect to the diagnostic sensitivity of the pleural biopsies performed with an Abrams' needle. For instance, in a recent study, the diagnostic sensitivities for pleural tuberculosis and malignant pleural pathologies were 89.5 and $91.7 \%$, respectively, when biopsies were performed under US guidance using an Abrams' needle [28]. In a previous series reported by our group, the success rate of CT-guided biopsy in patients with malignant mesothelioma was $83 \%$ [18]; the success rate was $87 \%$ in the aforementioned study that randomized patients to MT [6].

We think that there are 4 potential causes for the lower success rate with US-CNPB than with CT-ANPB. These are as follows: (1) when performing a Tru-Cut nee- dle biopsy, although generally multiple samples are obtained, biopsy sampling is frequently carried out in a single direction. On the other hand, an Abrams' needle allows sampling from different sites using rotations in different directions depending on the site of entry. (2) In Tru-Cut biopsies, the needle is mostly inserted into the pleura using a tangential angle, which may potentially limit the depth of sampling toward the submesothelial tissue. This may cause certain diagnostic challenges for a histopathologist, who would have to decide whether submesothelial invasion is present or not, as an important diagnostic criterion for malignant lesions. In a certain proportion of our patients randomized to study arms where both techniques were utilized, no pleural lesions or pleural thickening could be detected on CT scans or US images. This problem, i.e. inadequate sampling in terms of submesothelial depth, might have been an important issue for the cutting needle. (3) Since CT scans provide a total view of the thorax, they might have provided more useful information in terms of the choice of the point of entry, and also in terms of propagating the needle toward the lesion or toward the areas with pleural thickening, when compared to the more local image provided by the US. (4) In our study, the 'free-hand technique' was used in all patients undergoing US-CNPB (in all patients with pleural effusion regardless of the presence of pleural lesions), both due to our study purposes and also due to the characteristics of the patient groups. This technique might have given us a lower diagnostic efficacy than US$\mathrm{CNPB}$ in a limited number of our patients. The same issue was also mentioned by Koegelenberg et al. [24]. However, this latter issue warrants further, randomized, prospective trials for clarification.

The above-mentioned results suggest that not only the accuracy of the site of the pleural sampling is important in establishing a histopathological diagnosis but also the depth of the pleural/tumoral tissue. This conclusion has also been expressed by other authors, who have stated that the diagnostic sensitivity of image-guided biopsy was higher in the presence of pleural thickness $>10 \mathrm{~mm}$, pleural nodularity, pleural-based mass lesions, and solid pleural tumors $[10,15,29,30]$. For instance, in 10 patients with mesothelioma who exhibited pleural lesion thicknesses $>20 \mathrm{~mm}$, US-assisted Tru-Cut biopsy exhibited a diagnostic sensitivity of $100 \%$ [10]. Of 21 patients with mesothelioma, the results of percutaneous, image-guided cutting-needle biopsies were positive in $18(86 \%)$ patients [22]. In the present study, both samples using each needle were adequate for histopathological examination in more than $90 \%$ of the cases. However, for an accurate diagnosis, 
either pleural thickening or the appearance of the lesion was a significant parameter. In lesions analyzed with USCNPB, the diagnostic sensitivity for pleural thickness or lesions $<1$ and $\geq 1 \mathrm{~cm}$ was 42.9 and $80 \%$, respectively, a difference that was statistically significant. The same was true regarding CT-ANPB (56.5 vs. 93.7\%). These findings demonstrate that both needles obtained samples of adequate size and thickness in the presence of a prominent lesion; a significant decline in the adequacy of sampling was noted for both needles with thinner pleural lesions or lesions not visible on CT.

In patients with either no pleural effusion or a small pleural effusion, biopsies with Tru-Cut needles represented an excellent pleural biopsy alternative, with a reported diagnostic sensitivity for US-assisted pleural biopsy of approximately $80 \%[20,30]$.

Regarding complication rates, both methods proved safe among our patients. Other publications regarding image-guided biopsies have also reported low complication rates $[10,18,19,24]$.

Randomized evidence suggests that a blind pleural biopsy should not be used for the diagnosis of pleural disease except where pleural tuberculosis is endemic $[6,7$, $23,31]$. The choice between image-guided and thoraco- scopic biopsy is largely dependent on both the effusion size and the appearance on CT. Therefore, the appropriateness of a particular technique for a specific patient should be sought, as opposed to the superiority of a particular technique compared with alternatives [3].

We recommend that CT-ANPB be used as a first-line diagnostic evaluation in the event of a negative cytological investigation of pleural effusion in cases characterized by pleural thicknesses or pleural lesions $\geq 1 \mathrm{~cm}$ as noted via thoracic CT. US-CNPB should be used in cases in which only pleural thickening with no pleural effusion or a small pleural effusion is noted for which an Abrams' needle is inappropriate. Among patients exhibiting only fluid on CT or a pleural thickness $<1 \mathrm{~cm}$, we recommend that MT should be the primary diagnostic method utilized. It may be useful to evaluate these suggestions in future, prospective, multicenter studies.

\section{Financial Disclosure and Conflicts of Interest}

The authors report that no conflict of interest exists with any companies/organizations whose products or services may be discussed in this article.

\section{References}

1 Hooper C, Lee YC, Maskell N; BTS Pleural Guideline Group: Investigation of a unilateral pleural effusion in adults: British Thoracic Society Pleural Disease Guideline 2010. Thorax 2010;65(suppl 2):ii4-ii17.

$\checkmark 2$ Prakash UB, Reiman HM: Comparison of needle biopsy with cytologic analysis for the evaluation of pleural effusion: analysis of 414 cases. Mayo Clin Proc 1985;60:158-164

3 Rahman NM, Gleeson FV: Image-guided pleural biopsy. Curr Opin Pulm Med 2008;14: 331-336.

4 Rahman NM, Ali NJ, Brown G, et al: Local anaesthetic thoracoscopy: British Thoracic Society Pleural Disease Guideline 2010. Thorax 2010;65(suppl 2):ii54-ii60.

5 Benamore RE, Scott K, Richards CJ, Entwisle JJ: Image-guided pleural biopsy: diagnostic yield and complications. Clin Radiol 2006;61: 700-705.

6 Metintas M, Ak G, Dundar E, et al: Medical thoracoscopy versus CT scan-guided Abrams pleural needle biopsy for diagnosis of patients with pleural effusions: a randomized, controlled trial. Chest 2010;137:1362-1368.
7 Koegelenberg CFN, Bolliger CT, Theron J, et al: Direct comparison of the diagnostic yield of ultrasound-assisted Abrams and Tru-Cut needle biopsies for pleural tuberculosis. Tho$\operatorname{rax} 2010 ; 65: 857-862$.

8 Matin TN, Gleeson FV: Interventional radiology of pleural diseases. Respirology 2011;16: 419-429.

9 Hallifax RJ, Corcoran JP, Ahmed A, et al: Physician-based ultrasound-guided biopsy for diagnosing pleural disease. Chest 2014;146: 1001-1006.

10 Diacon AH, Schuurmans MM, Theron J, Schubert PT, Wright CA, Bolliger CT: Safety and yield of ultrasound-assisted transthoracic biopsy performed by pulmonologists. Respiration 2004;71:519-522.

11 Koegelenberg CFN, von Groote-Bidlingmaier F, Bolliger CT: Transthoracic ultrasonography for the respiratory phsyician. Respiration 2012;84:337-350.

12 Dixon G, de Fonseka D, Maskell N: Pleural controversies: image guided biopsy versus thoracoscopy for undiagnosed pleural effusions? J Thorac Dis 2015;6:1041-1051.

13 Blank W: International chest sonography; in Mathis G (ed): Chest Sonography. Berlin, Springer, 2008, pp 187-188.
14 Koegelenberg CFN, Diacon AH, Bolliger CT: Transthoracic ultrasound of the chest wall, pleura and the peripheral lung; in Bolliger CT, Herth FJF, Mayo PH (eds): Clinical Chest Ultrasound. Basel, Karger, 2009, pp 22-23.

15 Koegelenberg CF, Diacon AH: Pleural controversy: close needle pleural biopsy or thoracoscopy - which first? Respirology 2011;16: 738-746.

16 Chang DB, Yang PC, Luh KT, Kuo SH, Yu CJ: Ultrasound-guided pleural biopsy with TruCut needle. Chest 1991;100:1328-1333.

17 Cao YY, Fan N, Xing F, Xu LY, Qu YJ, Liao MY: Computed tomography-guided cutting needle pleural biopsy: accuracy and complications. Exp Ther Med 2015;9:262-266.

18 Metintas M, Ozdemir N, Isiksoy S, et al: CTguided pleural needle biopsy in the diagnosis of malignant mesothelioma. J Comput Assist Tomogr 1995;19:370-374.

19 Adams RF, Gleeson FV: Percutaneous imageguided cutting-needle biopsy of the pleura in the presence of a suspected malignant effusion. Radiology 2001;219:510-514.

20 Koegelenberg CFN, Diacon AH: Image-guided pleural biopsy. Curr Opin Pulm Med 2013; 19:368-373. 
21 Qureshi NR, Gleeson FV: Imaging of pleural disease. Clin Chest Med 2006;27:193-13.

22 Adams RF, Gray W, Davies RJ, Gleeson FV: Percutaneous image-guided cutting needle biopsy of the pleura in the diagnosis of malignant mesothelioma. Chest 2001;120:17981802.

23 Rezk NASA, Aly NYA, El-Hadidy TA, Dasthi $\mathrm{K}$ : CT-guided biopsy versus conventional Abrams' needle biopsy in malignant pleural effusion. Egypt J Chest Dis Tuberc 2015;64: 405-409.

24 Koegelenberg CFN, Irusen EM, von GrooteBidlingmaier F, Bruwer JW, Batubara EM, Diacon AH: The utility of ultrasound-guided thoracentesis and pleural biopsy in undiagnosed pleural exudates. Thorax 2015;70:995997.
25 Vorster MJ, Allwood BW, Koegelenberg FC: Transthoracic ultrasonography for clinicians. Euroasian J Pulmonol 2015;17:1-9.

26 Stigt JA, Groen HJ: Percutaneous ultrasonography as imaging modality and sampling guide for pulmonologists. Respiration 2014 87:441-451.

27 Botana Rial M, Briones Gómez A, Ferrando Gabarda JR, et al: Tru-cut needle pleural biopsy and cytology as the initial procedure in the evaluation of pleural effusion. Arch Bronconeumol 2014;50:313-317.
28 Botana-Rial M, Leiro-Fernández V, RepresasRepresas C, et al: Thoracic ultrasound-assisted selection for pleural biopsy with Abrams needle. Respir Care 2013;58:1949-1954.

29 Qureshi NR, Rahman NM, Gleeson FV: Thoracic ultrasound in the diagnosis of malignant pleural effusion. Thorax 2009;64:139-143.

30 Stigt JA, Boers JE, Groen HJ: Analysis of 'dry' mesothelioma with ultrasound guided biopsies. Lung Cancer 2012;78:229-233.

31 Maskell NA, Gleeson FV, Davies RJ: Standard pleural biopsy versus CT-guided cutting-needle biopsy for diagnosis of malignant disease in pleural effusions: a randomised controlled trial. Lancet 2003;19;361:1326-1330. 